\title{
Discriminating Between Log-Logistic and Rayleigh Distributions
}

\author{
B. Srinivasa Rao \\ Department of Mathematics \\ R.V.R \& J.C College of Engineering \\ Guntur-522 019, A.P, India \\ boyapatisrinu@yahoo.com \\ R.R.L. Kantam \\ Department of Statistics \\ Acharya Nagarjuna University \\ Guntur-522 010, A.P, India. \\ kantam_rrl@rediffmail.com
}

\begin{abstract}
Test statistics based on likelihood function, order statistics, population quantiles are suggested to discriminate between log-logistic distribution and Rayleigh distribution. Because of non tractability of their exact sampling distributions, the percentiles of the proposed test statistics are tabulated with the help of simulated sampling distributions of the test statistics. The power of the test statistics are also tabulated and a comparative study w.r.t. the powers for a given sample and the level of significance are worked out.
\end{abstract}

Keywords and phrases: Log-logistic distribution (LLD), Rayleigh distribution (RD), Likelihood function, Order statistics, Quantiles, average run length (ARL), Power of the test.

\section{Introduction}

The probability density function (pdf) of Log-logistic distribution with scale parameter $\sigma$ and shape parameter $\beta$ is given by

$$
f(x, \beta, \sigma)=\frac{\frac{\beta}{\sigma}\left(\frac{x}{\sigma}\right)^{\beta-1}}{\sigma\left(1+\left(\frac{x}{\sigma}\right)^{\beta}\right)^{2}}, \quad x>0, \sigma>0, \beta>1
$$

Its cumulative distribution function (cdf) is

$$
F(x, \beta, \sigma)=\frac{\left(\frac{x}{\sigma}\right)^{\beta}}{1+\left(\frac{x}{\sigma}\right)^{\beta}}, \quad x>0, \sigma>0, \beta>1
$$

The probability density function (pdf) of Rayleigh distribution with scale parameter $\sigma$ is given by

$$
f(x, \sigma)=\frac{x}{\sigma^{2}} e^{\frac{-x^{2}}{2 \sigma^{2}}}, \quad x>0, \sigma>0
$$


Its cumulative distribution function (cdf) is

$$
F(x, \sigma)=1-e^{\frac{-x^{2}}{\sigma^{2}}}, \quad x>0, \sigma>0
$$

Many people have studied the importance of these two distributions as life testing models. In fact inferential properties of Rayleigh distribution are simple, efficient and mathematically tractable, where as it is not the same case with log logistic distribution. We are therefore motivated to study whether Rayleigh distribution is a reason- able alternative to log-logistic distribution at least for the sake of adopting the analytical, powerful inferential characteristics of Rayleigh distribution for the data following log logistic distribution. With this backdrop we suggested three different test statistics to discriminate log logistic distribution (LLD) and Rayleigh distribution (RD). The research works on LLD and RD of recent origin include, Kantam et al. (2001), Kantam and Srinivasa Rao (2002), Gupta and Kundu (2004), Kantam and Srinivasa Rao (2004), Gupta et al (2005), Kantam et al. (2006), Kantam et al. (2007), Sultan (2007), Rosaiah et al (2007), Rosaiah et al. (2007), Srinivasa Rao and Kantam (2010). The rest of the paper is organized as follows. The proposed test statistics based on likelihood function, order statistics, population quantiles are respectively explained in Section 2, Section 3 and Section 4 along with their percentiles and power values. A comparative study of the three statistics with respect to their powers is presented in Section 5.

\section{Test statistics based on likelihood ratio}

Let us consider LLD with shape parameter 3 and scale parameter 1 as the null population say $\left(P_{0}\right)$, the RD with scale parameter 1 is considered as the alternative population say $\left(P_{1}\right)$. If a random sample of $x_{1}, x_{2}, \ldots, x_{n}$ is drawn from $P_{0}$ evaluate the estimation of the parameters of $P_{0}$ using the given sample of $x_{1}, x_{2}, \ldots, x_{n}$. Let $\hat{P}_{0}$ denote the value of the likelihood function of the sample $x_{1}, x_{2}, \ldots, x_{n}$ w.r.t. to null population, at the estimates of its parameters using $x_{1}, x_{2}, \ldots, x_{n}$. Let $\hat{P}_{1}$ denote the value of likelihood function w.r.t to alternative population, the estimates of $P_{1}$ using the sample $x_{1}, x_{2}, \ldots, x_{n}$. The ratio $\frac{L_{1}}{L_{0}}$ in a way represents the ratio of the likelihood of $P_{1}$ to that of $P_{0}$ with a sample drawn from $P_{0}$. Therefore $\frac{P_{1}}{P_{0}}$ is the ratio of a smallest probability to a larger probability and hence is expected to be small. The null hypothesis " $\mathrm{H}_{0}$ : The sample is drawn from $\mathrm{P}_{0}$ " can be tested using the percentiles of the likelihood ratio $\frac{L_{1}}{L_{0}}$. Therefore statistics $T_{1}=\frac{L_{1}}{L_{0}}$ can be taken as test statistics to test the above null hypothesis. Since the distribution of $\frac{L_{1}}{L_{0}}$ is not analytical, we have 
computed the percentiles of empirical sampling distributions of $T_{1}=\frac{L_{1}}{L_{0}}$ with the help of 10,000 simulation runs of sample size $n=2, . ., 10$ are given in table 1 .

Table 1: Percentiles of sampling distributions of $T_{1}=\frac{L_{1}}{L_{0}}$

\begin{tabular}{|c|c|c|c|c|c|c|c|c|}
\hline $\mathrm{n}$ & 0.99 & 0.975 & 0.95 & 0.90 & 0.10 & 0.05 & 0.025 & 0.01 \\
\hline 2 & 1.7717 & 1.3158 & 1.2315 & 1.1206 & 0.4558 & 0.4532 & 0.4520 & 0.1769 \\
3 & 1.5436 & 1.2517 & 0.9609 & 0.8393 & 0.3102 & 0.3061 & 0.2314 & 0.0132 \\
4 & 1.4393 & 1.1596 & 0.8482 & 0.6369 & 0.2113 & 0.2078 & 0.0983 & 0.0014 \\
5 & 1.0590 & 0.8585 & 0.6987 & 0.4994 & 0.1454 & 0.1407 & 0.0515 & 0.0000 \\
6 & 0.9915 & 0.8405 & 0.6151 & 0.4121 & 0.0990 & 0.0916 & 0.0084 & 0.0000 \\
7 & 0.9656 & 0.5941 & 0.4558 & 0.3334 & 0.0692 & 0.0534 & 0.0041 & 0.0000 \\
8 & 0.7245 & 0.5132 & 0.3883 & 0.2715 & 0.0484 & 0.0264 & 0.0019 & 0.0000 \\
9 & 0.7367 & 0.4417 & 0.3068 & 0.2165 & 0.0330 & 0.0141 & 0.0004 & 0.0000 \\
10 & 0.4876 & 0.3649 & 0.2467 & 0.1772 & 0.0228 & 0.0062 & 0.0002 & 0.0000 \\
\hline
\end{tabular}

The power of the test statistics $T_{1}$ is also tabulated for two levels of significance (5\% and $10 \%$ ) at sample sizes $\mathrm{n}=2, . ., 10$ by simulating samples from $P_{1}$ and using the values of $T_{1}$. The count of $T_{1}$ value that fall beyond the table values of Table 1 shall speak of the power of $T_{1}$. These are given in table 2 .

\section{Table 2: Power of $T_{1}$}

\begin{tabular}{|c|c|c|}
\hline $\mathrm{n}$ & 0.99 & 0.95 \\
\hline 2 & 0.7230 & 0.7420 \\
3 & 0.9170 & 0.9350 \\
4 & 0.9410 & 0.9760 \\
5 & 0.9830 & 0.9920 \\
6 & 0.9910 & 0.9960 \\
7 & 0.9960 & 0.9970 \\
8 & 0.9980 & 0.9980 \\
9 & 0.9980 & 0.9990 \\
10 & 0.9980 & 1.000 \\
\hline
\end{tabular}

These tables indicate that even with the help of a small sample of size as small as $n=2$ the power remains to be at more than $80 \%$ and is increasing as $\mathrm{n}$ increases. It is therefore concluded the $T_{1}$ statistic proposed in this section can discriminate between the null and alternative population with a high power values as given in table 2 . 


\section{Test statistics based on Order Statistics}

Let $x_{1}, x_{2}, \ldots, x_{n}$ denote sample from Log logistic distribution. The correlation-type goodness-of-fit test in this case using order statistics can be formed as

$$
T_{2}=\frac{\sum_{i=1}^{n} x_{i} \alpha_{i}}{\sqrt{\sum_{i=1}^{n} x_{i}^{2} \sum_{i=1}^{n} \alpha_{i}^{2}}}
$$

where $\alpha_{i}$ is expected value of $\mathrm{i}^{\text {th }}$ order statistic in a sample of size $\mathrm{n}$ drawn from $P_{0}$ and $x_{i}$ is the $\mathrm{i}^{\text {th }}$ order sample observation $\alpha_{i}$ can be borrowed from Balakrishnan and Malik (1987). This statistic represents the correlation between $x_{i}$ and $\alpha_{i}$, $i=1,2, \ldots, n$. The statistic $T_{2}$ is simulated through Monte-Carlo method based on 10,000 simulations. Table 3 represents the percentiles of $T_{2}$ for sample sizes $n=2, . ., 10$ and various levels of significance.

Table 3: Percentiles of $T_{2}$ based on order statistics

\begin{tabular}{|c|c|c|c|c|c|c|c|c|}
\hline $\mathrm{n}$ & 0.99 & 0.975 & 0.95 & 0.90 & 0.10 & 0.05 & 0.025 & 0.01 \\
\hline 2 & 1.0000 & 1.0000 & 0.9997 & 0.9985 & 0.9544 & 0.9255 & 0.9499 & 0.9490 \\
3 & 0.9993 & 0.9985 & 0.9972 & 0.9950 & 0.9408 & 0.9349 & 0.9307 & 0.9243 \\
4 & 0.9977 & 0.9965 & 0.9952 & 0.9929 & 0.9339 & 0.9271 & 0.9210 & 0.9101 \\
5 & 0.9970 & 0.9954 & 0.9939 & 0.9914 & 0.9290 & 0.9220 & 0.9154 & 0.8989 \\
6 & 0.9965 & 0.9950 & 0.9931 & 0.9904 & 0.9260 & 0.9185 & 0.9119 & 0.8893 \\
7 & 0.9960 & 0.9946 & 0.9929 & 0.9903 & 0.9238 & 0.9166 & 0.9087 & 0.8796 \\
8 & 0.9957 & 0.9941 & 0.9923 & 0.9897 & 0.9227 & 0.9144 & 0.9060 & 0.8730 \\
9 & 0.9954 & 0.9939 & 0.9921 & 0.9895 & 0.9217 & 0.9124 & 0.9031 & 0.8701 \\
10 & 0.9951 & 0.9936 & 0.9919 & 0.9893 & 0.9215 & 0.9114 & 0.9016 & 0.8557 \\
\hline
\end{tabular}

As we can see from Table 3 , the percentiles of $T_{2}$ increase as the sample size increases as well as the significance level increases. The power of the test statistics $T_{2}$ is also tabulated for two levels of significance $(5 \%$ and $10 \%)$ at sample sizes by $n=2, . ., 10$ simulating samples from $P_{1}$ and using the values of $T_{2}$. The count of $T_{2}$ value that fall beyond the table values of Table 3 shall speak of the power of $\mathrm{T}_{2}$. These are given in table 4 . 
Table 4: Power of $T_{2}$

\begin{tabular}{|c|c|c|}
\hline $\mathrm{n}$ & 0.99 & 0.95 \\
\hline 2 & 0.9465 & 0.8867 \\
3 & 0.9578 & 0.9064 \\
4 & 0.9689 & 0.9278 \\
5 & 0.9746 & 0.9851 \\
6 & 0.9805 & 0.9529 \\
7 & 0.9869 & 0.9674 \\
8 & 0.9913 & 0.9729 \\
9 & 0.9952 & 0.9811 \\
10 & 0.9968 & 0.9859 \\
\hline
\end{tabular}

\section{Test statistics based on Quantiles}

Let $x_{1}, x_{2}, \ldots, x_{n}$ denote sample from Log logistic distribution. The correlation-type goodness-of-fit test in this case using percentiles can be formed as

$$
T_{3}=\frac{\sum_{i=1}^{n} Z_{i} v_{i}}{\sqrt{\sum_{i=1}^{n} Z_{i}^{2} \sum_{i=1}^{n} v_{i}^{2}}}
$$

This statistic represents the correlation between $x_{i}$ and $v_{i}, i=1,2, \ldots, n$ where $v_{i}$ is the $\mathrm{i}^{\text {th }}$ quantile of the null population. The statistic $T_{3}$ is simulated through MonteCarlo method based on 10,000 simulations. Table 3 represents the percentiles of $T_{3}$ for sample sizes $n=2(1) 10$ and various levels of significance.

Table 5: Percentiles of $T_{3}$ based on quantiles

\begin{tabular}{|c|c|c|c|c|c|c|c|c|}
\hline $\mathrm{n}$ & 0.99 & 0.975 & 0.95 & 0.90 & 0.10 & 0.05 & 0.025 & 0.01 \\
\hline 2 & 1.0000 & 0.9999 & 0.9998 & 0.9992 & 0.9760 & 0.9618 & 0.9435 & 0.9220 \\
3 & 0.9998 & 0.9991 & 0.9982 & 0.9964 & 0.9636 & 0.9422 & 0.9171 & 0.8792 \\
4 & 0.9986 & 0.9978 & 0.9968 & 0.9952 & 0.9570 & 0.9311 & 0.9001 & 0.8616 \\
5 & 0.9980 & 0.9969 & 0.9957 & 0.9941 & 0.9523 & 0.9226 & 0.8858 & 0.8451 \\
6 & 0.9973 & 0.9963 & 0.9950 & 0.9932 & 0.9472 & 0.9133 & 0.8793 & 0.8338 \\
7 & 0.9971 & 0.9958 & 0.9946 & 0.9926 & 0.9442 & 0.9094 & 0.8709 & 0.8207 \\
8 & 0.9967 & 0.9955 & 0.9942 & 0.9922 & 0.9408 & 0.9055 & 0.8686 & 0.8136 \\
9 & 0.9964 & 0.9953 & 0.9938 & 0.9919 & 0.9381 & 0.9013 & 0.8631 & 0.8105 \\
10 & 0.9961 & 0.9949 & 0.9936 & 0.9915 & 0.9357 & 0.8985 & 0.8592 & 0.7923 \\
\hline
\end{tabular}


As we can see from Table 5, the percentile points of $T_{3}$ increase as the sample size increases as well as the significance level increases. The power of the test statistics $T_{3}$ is also tabulated for two levels of significance (5\% and 10\%) at sample sizes $n=2(1) 10$ by simulating samples from $P_{1}$ and using the values of $T_{3}$. The count of $T_{3}$ value that fall beyond the table values of table 5 shall speak of the power of $T_{3}$. These are given in Table 6.

\section{Table 6: Power of $T_{3}$}

\begin{tabular}{|c|c|c|}
\hline $\mathrm{n}$ & 0.99 & 0.95 \\
\hline 2 & 0.9454 & 0.8843 \\
3 & 0.9344 & 0.8621 \\
4 & 0.9419 & 0.8729 \\
5 & 0.9353 & 0.8798 \\
6 & 0.9363 & 0.8759 \\
7 & 0.9382 & 0.8706 \\
8 & 0.9462 & 0.8766 \\
9 & 0.9499 & 0.8871 \\
10 & 0.9551 & 0.8914 \\
\hline
\end{tabular}

\section{Comparative study}

The powers given in tables 2, 4 and 6 for the test statistics $T_{1}, T_{2}$, and $T_{3}$, respectively indicate that all the three statistics discriminate between log-logistic and Rayleigh distributions quite significantly. This is true even in small samples of size as small as 3. But the statistics based on the formulae $T_{1}$ and $T_{2}$ require a sound method of estimator or specially tabulated expected values of order statistics of samples drawn from log-logistic distribution. However, the statistic $T_{3}$ is based on the population quantiles of log-logistic distribution corresponding to a given sample of size $\mathrm{n}$ that can be easily calculate by inverting the analytical expression of the population distribution function of log-logistic distribution. This inversion does not require any specific tables and accordingly simpler. We can see that the coverage probability based on order statistics $\left(T_{2}\right)$ is more powerful than those on likelihood ratio and population quantiles.

\section{References}

1. Balakrishnan. N. and Malik. H.J. (1987). Moments of order statistics from truncated log-logistic distribution, Journal of Statistical Planning and Inference, 17, 251-267.

2. Gupta. D.R. and Kundu. D. (2004). Discriminating between gamma and generalized exponential distributions, Journal of Statistical computation and Simulation, 74(2), 107-121. 
3. Gupta. D.R., Kundu. D. and Manglic. A. (2005). Discriminating between log normal and generalized exponential distributions, Journal of Statistical planning and Inference, 127, 213-227, 2005.

4. Kantam. R.R.L., Rosaiah. K. and Srinivasa Rao. G. (2001). Acceptance sampling based on life tests Log-logistic model, Journal of Applied Statistics, 28(1), 121-128.

5. Kantam. R.R.L. and Srinivasa Rao. G. (2002). Log-logistic distribution: Modified maximum likelihodd estimation, Gujarat Statistical Review, 29 (1 \& 2), 25-36.

6. Kantam. R.R.L. and Srinivasa Rao. G. (2004). A note on point estimation of system reliability exemplified for the log-logistic distribution, Economic Quality Control, 19 (2), 197-204.

7. Kantam. R.R.L., Vasudeva Rao. A. and Srinivasa Rao. G. (2006). Control charts for Log-logistic distribution, Economic Quality Control, 21(1), 77-86.

8. Kantam. R.R.L., Srinivasa Rao. G. and Sriram. B. (2007). On stressstrength reliability model on log-logistic distribution, International Journal of Agricultural Statistical Science, 3(1), 1-6.

9. Rosaiah. K., Kantam. R.R.L., Prasad. A.R.S. and Srinivasa Rao. G. (2007). Log-logistic model: Approximations to ML method, Statistical Association, 45(1), 109-122.

10. Rosaiah. K., Kantam. R.R.L. and Poleswara Rao. G. (2007a). Reliability estimation from damaged data in Rayleigh distribution, International Journal of Agricultural Statistical Science, 3(1), 281-287.

11. Srinivasa Rao. G. and Kantam. R.R.L. (2010). Acceptance sampling plans from truncated life tests based on log-logistic distribution for percentiles, Economic Quality Control, 25(2), 153-167.

12. Sultan. K.S. (2007). Order Statistics from the Generalized Exponential Distribution and Applications, Communications in Statistics - Theory and Methods, 36, 1409-1418. 